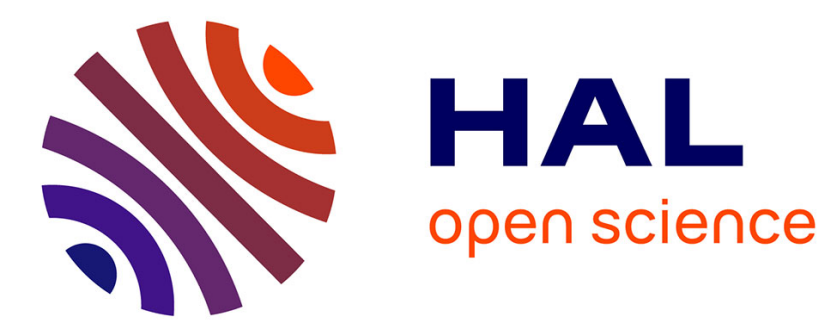

\title{
Homogenization-Based Predictions for Texture Evolution in Halite
}

P. Gilormini, Y. Liu, Pedro Ponte Castañeda

\section{To cite this version:}

P. Gilormini, Y. Liu, Pedro Ponte Castañeda. Homogenization-Based Predictions for Texture Evolution in Halite. IUTAM Symposium on Computational Mechanics of Solid Materials at Large Strains, Aug 2001, Stuttgart, Germany. pp.269-278, 10.1007/978-94-017-0297-3_24 . hal-02266326

\section{HAL Id: hal-02266326 \\ https://hal.science/hal-02266326}

Submitted on 13 Aug 2019

HAL is a multi-disciplinary open access archive for the deposit and dissemination of scientific research documents, whether they are published or not. The documents may come from teaching and research institutions in France or abroad, or from public or private research centers.
L'archive ouverte pluridisciplinaire HAL, est destinée au dépôt et à la diffusion de documents scientifiques de niveau recherche, publiés ou non, émanant des établissements d'enseignement et de recherche français ou étrangers, des laboratoires publics ou privés. 


\title{
Homogenization-Based Predictions for Texture Evolution in Halite
}

\author{
P. Gilormini \\ Laboratoire de Mécanique et Technologie, ENS de Cachan-CNRS-Université de Paris 6 \\ 94235 Cachan, France
}

Pierre.Gilormini@Imt.ens-cachan.fr

Y. Liu, P. Ponte Castañeda

Department of Mechanical Engineering and Applied Mechanics, University of Pennsylvania 220 S. 33rd. Street, Philadelphia, PA 19104-6315, U.S.A

yiliu2@seas.upenn.edu, ponte@seas.upenn.edu

\begin{abstract}
The "variational" homogenization method developed by deBotton and Ponte Castañeda [2] is used here to predict texture development in halite polycrystals at room and high temperatures accounting for hardening and grain shape changes. The new predictions are compared with those of the Taylor and "tangent" model of Molinari et al. [5] for uniaxial tension and compression. The predictions of the "variational" model are found to be intermediate between the Taylor and "tangent" predictions, although not too different from either, as a consequence of the relatively high isotropy of the halite single crystal grains.
\end{abstract}

Keywords: Polycrystals, plasticity, texture evolution, halite.

\section{Introduction}

Halite $(\mathrm{NaCl})$ is a polycrystalline aggregate of ionic single crystals with cubic structure. In this study, the crystals are assumed to deform by crystallographic slip only, thus neglecting recrystallization and other potential deformation mechanisms. For halite, three families of slip systems are usually activated; they are the types A (6 $\{110\}\langle 110\rangle$ systems $), \mathrm{B}(6\{100\}\langle 110\rangle$ systems $)$ and $\mathrm{C}(12\{111\}\langle 110\rangle$ systems; the same as in fcc crystals) families. A power-law relation is assumed to hold between the resolved shear stress and the slip rate on a given system, which is a reasonable approximation for halite at least at high temperature according to Carter and Heard [1] and Heard [3]. For simplicity, a value of $n=7$ will be assumed for all slip families in the high-temperature simulations, which provides a reasonably good fit with the results of Heard [3] at $400^{\circ} \mathrm{C}$. On the other hand, an exponent of $n=10$ is used for the simulations at room temperature, which was close to the value of 10.5 reported in Carter and Heard [1] for the A systems (no values were given for the B or C systems). In addition, a hardening model incorporating latent hardening and saturation 


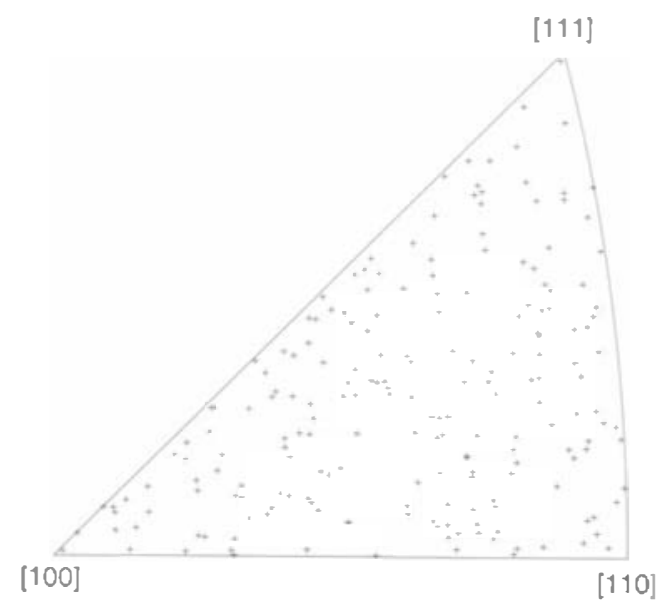

Figure 1. Inverse pole figure of the 200-grain initial random texture.

values was used, as described in more detail later, to fit the experimental data for halite single crystals at both room and high temperature.

An initially random texture of 200 grains was used (Figure 1) for all simulations, having verified that use of other initially random distributions of 200 orientations had little effect on the results. In this work, we will make use of the "variational" homogenization method of deBotton and Ponte Castaneda [2] and Nebozhyn et al. [6] to estimate the evolution of crystallographic texture in halite polycrystals, following the detailed proposal of Ponte Castaneda [9] to account for microstructure evolution. The results will be compared with the predictions of the Taylor estimate and "tangent" model of Molinari et al. [5].

\section{Formulation}

The halite polycrystals are assumed to occupy region in space $\Omega$ and can be approximated as $N$-phase composites, where each "phase" is defined as the subregion $\Omega^{(r)}(r=1, \cdots, N)$ occupied by all grains of a given orientation, as measured by a rotation tensor $\mathbf{Q}^{(r)}$ relative to a "reference" crystal with known orientation. Each grain is assumed to undergo viscous deformation on a set of $K(k=1, \cdots, K)$ preferred crystallographic slip systems, which are characterized by the second-order tensors

$$
\boldsymbol{\mu}_{(k)}^{(r)}=\frac{1}{2}\left(\mathbf{n}_{(k)}^{(r)} \otimes \mathbf{m}_{(k)}^{(r)}+\mathbf{m}_{(k)}^{(r)} \otimes \mathbf{n}_{(k)}^{(r)}\right),
$$

where $\mathbf{n}_{(k)}^{(r)}$ and $\mathbf{m}_{(k)}^{(r)}$ are the unit vectors normal to the slip plane and along the slip direction in the $k$ th system. 
Local behavior. For a given Cauchy stress $\sigma$, acting on the $k$ th slip system with orientation $r$, we introduce the resolved shear stresses $\tau_{(k)}^{(r)}=\boldsymbol{\sigma} \cdot \boldsymbol{\mu}_{(k)}^{(r)}$, so that the response of the grains with orientation $r$ may be written in the form

$$
\varepsilon=\frac{\partial u^{(r)}}{\partial \boldsymbol{\sigma}}(\boldsymbol{\sigma}), \quad u^{(r)}(\boldsymbol{\sigma})=\sum_{k=1}^{K} \phi_{(k)}\left(\tau_{(k)}^{(r)}\right),
$$

where $\varepsilon$ is the Eulerian strain rate and $u^{(r)}$ is the stress potential for the crystals with orientation $r$. In this last relation, the functions $\phi_{(k)}$, characterizing the slip potentials of the reference crystal, are assumed to be convex. Here, they will be chosen to be of the power-law type:

$$
\phi_{(k)}(\tau)=\frac{\dot{\gamma}_{0} \tau_{k}}{n+1}\left(\frac{|\tau|}{\tau_{k}}\right)^{n+1}
$$

where $0 \leq m=1 / n \leq 1, \tau_{k}>0$ and $\dot{\gamma}_{0}=1 /$ sec are the strain-rate sensitivity, reference stress of the $k$ th slip system and reference strain rate, respectively. Then, the slip strain rate $\dot{\gamma}$ in system $k$ is given by $\dot{\gamma}=\dot{\gamma}_{0}\left(|\tau| / \tau_{k}\right)^{n} \operatorname{sign}(\tau)$.

Effective behavior. Assuming that the size of of the typical grain is small relative to the size of the specimen under consideration, one defines volume averages $\langle$.$\rangle and \langle.\rangle^{(r)}$ over $\Omega$ and $\Omega^{(r)}$, respectively, so that theeffective response for the polycrystal may be written in the form

$$
\overline{\boldsymbol{\varepsilon}}=\frac{\partial \tilde{U}}{\partial \overline{\boldsymbol{\sigma}}}(\overline{\boldsymbol{\sigma}}), \quad \tilde{U}(\overline{\boldsymbol{\sigma}})=\min _{\boldsymbol{\tau} \in \mathcal{S}(\overline{\boldsymbol{\sigma}})} \sum_{r=1}^{N} c^{(r)}\left\langle u^{(r)}(\boldsymbol{\tau})\right\rangle^{(r)},
$$

where $\tilde{U}$ is the effective potential for the polycrystal, $\bar{\sigma}=\langle\boldsymbol{\sigma}\rangle$ and $\bar{\varepsilon}=<$ $\varepsilon>$ are the average stress and strain rate, the $c^{(r)}=\left\langle\chi^{(r)}\right\rangle$ characterize the texture of the polycrystal, and $\mathcal{S}$ is set of statically admissible stresses: $\mathcal{S}(\overline{\boldsymbol{\sigma}})=\{\boldsymbol{\tau}, \operatorname{div} \boldsymbol{\tau}=0$ in $\Omega, \boldsymbol{\tau} \mathbf{n}=\overline{\boldsymbol{\sigma}} \mathbf{n}$ on $\partial \Omega\}$.

Variational homogenization method. Following an analogous development by Ponte Castañeda [8] for $N$-phase composites with isotropic phases, deBotton and Ponte Castañeda [2] derived an alternative variational form for the effective potential (4) of the nonlinear polycrystal defined by (2), for some given set of slip potentials $\phi_{(k)}$. This variational representation involves a "linear comparison polycrystal" with phase potentials defined by

$$
u_{0}^{(r)}(\boldsymbol{\sigma})=\frac{1}{2} \boldsymbol{\sigma} \cdot \mathbf{M}^{(r)} \boldsymbol{\sigma}, \quad \mathbf{M}^{(r)}=2 \sum_{k=1}^{K} \alpha_{(k)}^{(r)} \boldsymbol{\mu}_{(k)}^{(r)} \otimes \boldsymbol{\mu}_{(k)}^{(r)} .
$$


Under appropriate hypotheses, a general bound for $\tilde{U}$ may be then obtained:

$$
\tilde{U}(\overline{\boldsymbol{\sigma}}) \geq \max _{\substack{\alpha_{(k)}^{(r)}>0, r=1, \ldots, N, k=1, \ldots, K}}\left\{\frac{1}{2} \overline{\boldsymbol{\sigma}} \cdot \tilde{\mathbf{M}}(\boldsymbol{\alpha}) \overline{\boldsymbol{\sigma}}-\sum_{r=1}^{N} \sum_{k=1}^{K} c^{(r)} V_{(k)}\left(\alpha_{(k)}^{(r)}\right)\right\},
$$

where

$$
V_{(k)}(\alpha)=\max _{\tau}\left\{\alpha|\tau|^{2}-\phi_{(k)}(\tau)\right\}
$$

serves to characterize the nonlinearity of the material behavior. Here also, $\alpha$ denotes the full set of positive slip compliances $\alpha_{(k)}^{(r)}$ and $\tilde{\mathbf{M}}$ is the effective compliance tensor of the linear comparison polycrystal with grain compliances $\mathbf{M}^{(r)}$, as defined by (5) in terms of the slip compliances $\boldsymbol{\alpha}$. As suggested by Nebozhyn et al. [6], use will be made of the standard self-consistent approximation to estimate the effective compliance tensor $\tilde{\mathbf{M}}$ of the linear comparison polycrystal.

If the maximum in (6) is attained at the stationarity condition, it follows that the effective constitutive relation for the polycrystal may be approximated as

$$
\overline{\boldsymbol{\varepsilon}}=\tilde{\mathbf{M}}\left(\hat{\boldsymbol{\alpha}} ; s^{(\alpha)}, \tau_{k}\right) \overline{\boldsymbol{\sigma}}
$$

where $\hat{\boldsymbol{\alpha}}$ now denotes the set of optimal $\alpha_{(k)}^{(r)}$ from (6). Here, the variables $s^{(\alpha)}$ and $\tau_{k}$ denote the instantaneous values of the microstructural variables characterizing the "state" of the crystallographic and morphological textures of the polycrystal, and of the reference slip stresses, respectively. In general, they evolve in time as a consequence of the finite deformation imposed on the polycrystal.

Microstructure evolution. For the "granular" microstructures of the viscoplastic polycrystals, it will be assumed that the distribution of the grains is controlled by the mean flow. In other words, the "shape" and "orientation" of the grains, characterizing the morphological texture, are assumed to be controlled by the average strain rate $\bar{\varepsilon}$ and spin $\bar{\omega}$ in the composite. This is required to preserve the integrity of the composite - for example, if every grain was to deform with the average strain and spin tensor in the grains, the composite would likely disintegrate, as each grain would in general be expected to have a different strain and spin field. In addition, it is also necessary to keep track of the orientation of the material within each grain, characterizing thecrystallographic texture. The evolution of this orientation distribution function will be assumed here to be controlled by the average $\operatorname{spin} \bar{\omega}^{(r)}$ of the material within the grains, as determined by the self-consistent estimates of the previous section for nonlinearly viscous polycrystals. Although a continuum distribution 
of orientations could be considered, for simplicity, only a discrete set of $N$ orientations, corresponding to $N$ different "phases," will be considered here. For more details, refer to Ponte Castañeda [9].

Slip systems hardening law. A reasonably good fit with the uniaxial extension data of Carter and Heard [1] for halite single crystals could be obtained with a hardening law incorporating latent hardening, as well as flow stress saturation levels. For each temperature, the sum of the squared differences with the 12 experimental points was minimized with respect to the 10 parameters of the model. Because of the nonuniqueness, and because the lowest minimum was not necessarily reasonable, a set of parameters has been selected that both gave good agreement with the measurements and used physically meaningful values (especially for latent hardening). This hardening law is described by the following relations:

$$
\dot{\tau}_{k}=\sum_{l=1}^{24} H_{k l}\left|\dot{\gamma}_{l}\right|
$$

where

$$
H_{k l}=h_{k}^{0}\left(\frac{\tau_{k}^{s a t}-\tau_{k}}{\tau_{k}^{s a t}-\tau_{k}^{0}}\right) Q_{k l} \quad(\text { no sum over } k)
$$

with $k=1, \ldots, 6$ for the A family, etc. The $Q_{k l}$ values define the effect of slip rate $\dot{\gamma}_{l}$ of system $l$ on the hardening of reference stress $\tau_{k}$ on system $k$. Here, a simple latent hardening model is used:

$$
Q_{k l}= \begin{cases}1 & \text { if systems } k \text { and } l \text { are coplanar } \\ q & \text { otherwise }\end{cases}
$$

This law leads to different hardening rates on the various families, and also on the various systems in each family. The hardening rate $H_{k l}$ equals either $h_{k}^{0}$ (self-hardening) or $q h_{k}^{0}$ (latent hardening) initially (when $\tau_{k}=\tau_{k}^{0}$ ), and it decreases to 0 when $\tau_{k}$ increases and tends to $\tau_{k}^{s a t}$, which corresponds to a stabilized value of $\tau_{k}$. This hardening law differs from one used, for instance, by Kalidindi et al. [4], where $H_{l k}$ replaces $H_{k l}$ (the function between $h_{k}$ and $Q_{k l}$ was also changed to a Voce law in the present work). Such a modification of the subscripts is already mentioned in Paquin et al. [7], but without comment. The reason for using $H_{k l}$ rather than $H_{l k}$ is for keeping the physical significance of the saturation value: when $\tau_{k}=\tau_{k}^{s a t}, H_{k l}$ is zero for all $l$, and consequently $\dot{\tau}_{k}=0$ in multislip conditions, whereas $\dot{\tau}_{k}$ can be nonzero if $H_{l k}$ is used in (10), which nullifies a column rather than a row of the $[H]$ matrix.

Although results are not shown for brevity, it was found that the values given in Table 1 for the 10 parameters involved in the hardening law gave good agreement with the experimental data. The fit was better for high temperature than for room temperature. The $\tau_{A}^{0}, \tau_{B}^{0}$ and $\tau_{C}^{0}$ values shown in Table 1 indicate that the single 
crystals will be almost isotropic at high temperature, as discussed above, at least initially. By contrast, some anisotropy is expected at room temperature.

Table 1. Halite single crystal parameters for a hardening law of the Voce type on each family of slip systems ( $h$ and $\tau$ values in MPa).

\begin{tabular}{ccc}
\hline Parameter & $25^{\circ} \mathrm{C}$ & $400^{\circ} \mathrm{C}$ \\
\hline$n$ & 10 & 7 \\
$q$ & 1.5 & 1.14 \\
$h_{A}^{0}$ & 170 & 13.0 \\
$h_{B}^{0}$ & 740 & 42.2 \\
$h_{C}^{0}$ & 45 & 21.5 \\
$\tau_{A}^{0}$ & 8.7 & 5.5 \\
$\tau_{B}^{0}$ & 20.7 & 6.0 \\
$\tau_{C}^{0}$ & 45.0 & 5.5 \\
$\tau_{A}^{\text {sat }}$ & 62 & 8.0 \\
$\tau_{B}^{\text {sat }}$ & 110 & 12.2 \\
$\tau_{C}^{\text {sat }}$ & 250 & 9.0 \\
\hline
\end{tabular}

\section{Textures predictions at $100 \%$ strain}

In this section, the above-described "variational" model is used to generate predictions for the crystallographic texture evolution of halite polycrystals. Following Wenk et al. [11], both uniaxial tension and compression are considered, and the two cases discussed above (i.e., room temperature and $400^{\circ} \mathrm{C}$ ) in the context of hardening are implemented. Comparisons are given with the Taylor model, as well as the "tangent" model. In this last connection, it should be noted that the "tangent" predictions shown here use the hypothesis that all the grains evolve with the same aspect ratio - in contrast with what was done by Wenk et al., but in agreement with what is done in our "variational" model.

Figures 2 and 3 show the inverse pole figures obtained in tension and compression, respectively. The general trend is a strong similitude between the results given by the "variational" and Taylor models, and some differences with the "tangent" model. For tension at room temperature, the "tangent" model predicts a sharp texture at $[100]$ and [111], whereas the "variational" model gives slightly lower values (but higher than the Taylor model). At high temperature (the more "isotropic" case), the three models give even closer results, with the above trends still present but much less pronounced. For compression (Figure 3), where textures are less sharp after a 100\% strain, the "tangent" model predicts a texture component at [100], but the other models do not. The results of the "variational" and Taylor models are quite similar globally, but exhibit lower values than those predicted by the "tangent" model. 

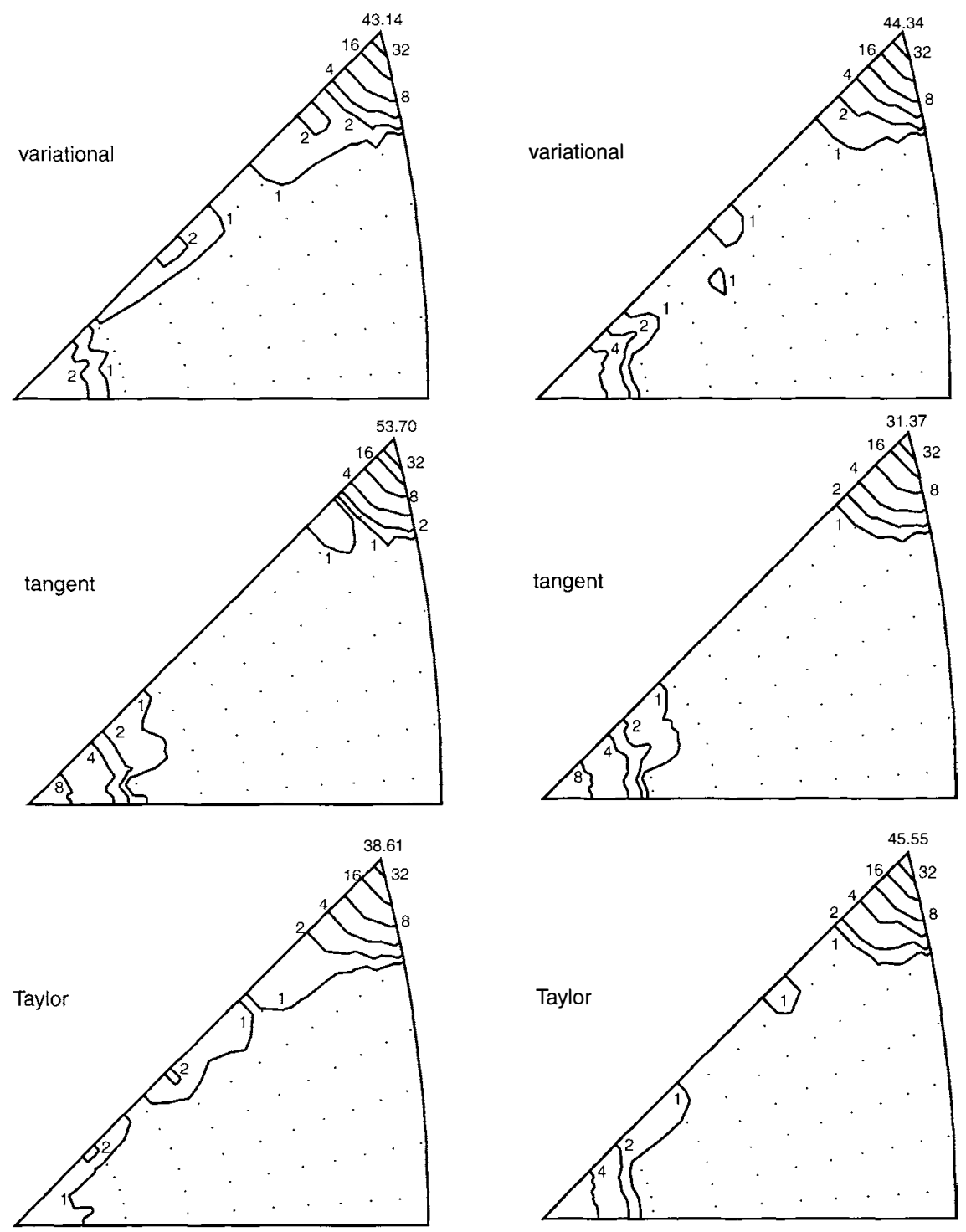

Figure 2. Inverse pole figures obtained after an axial strain of $100 \%$ applied under uniaxial tension at low $\left(25^{\circ} \mathrm{C}\right)$ and high $\left(400^{\circ} \mathrm{C}\right)$ temperatures. Comparison between the variational self-consistent, tangent self-consistent and Taylor models. Dotted areas are below the average level of 1 . 
$25^{\circ} \mathrm{C}$
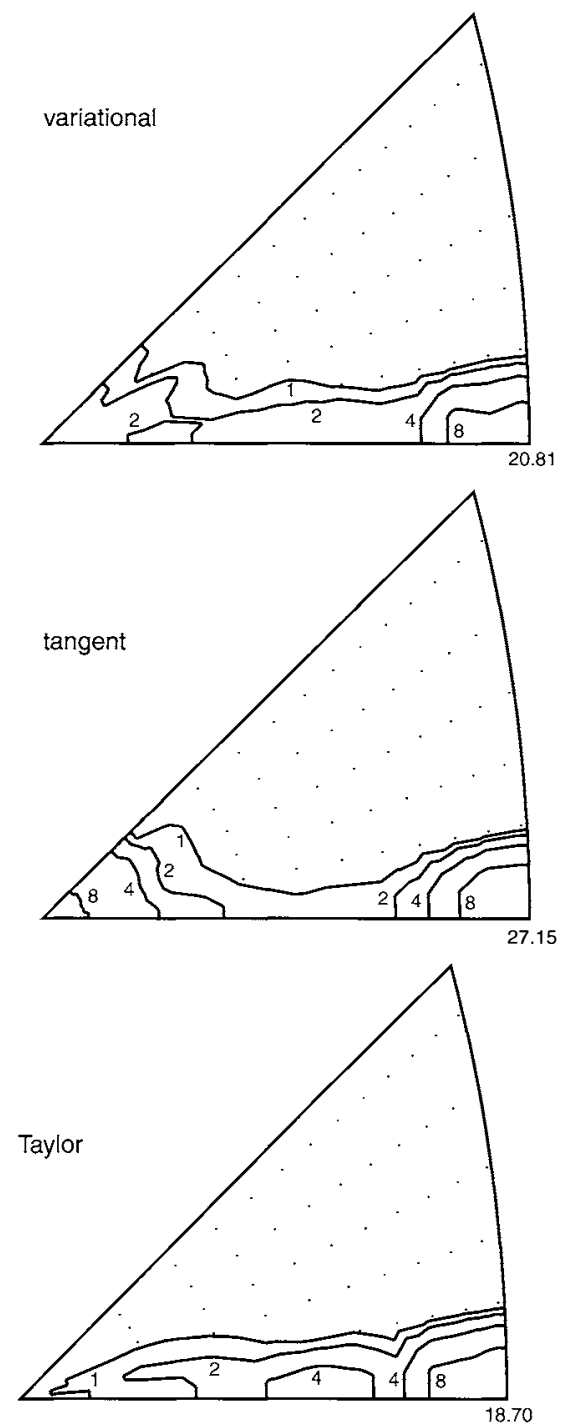

$400^{\circ} \mathrm{C}$
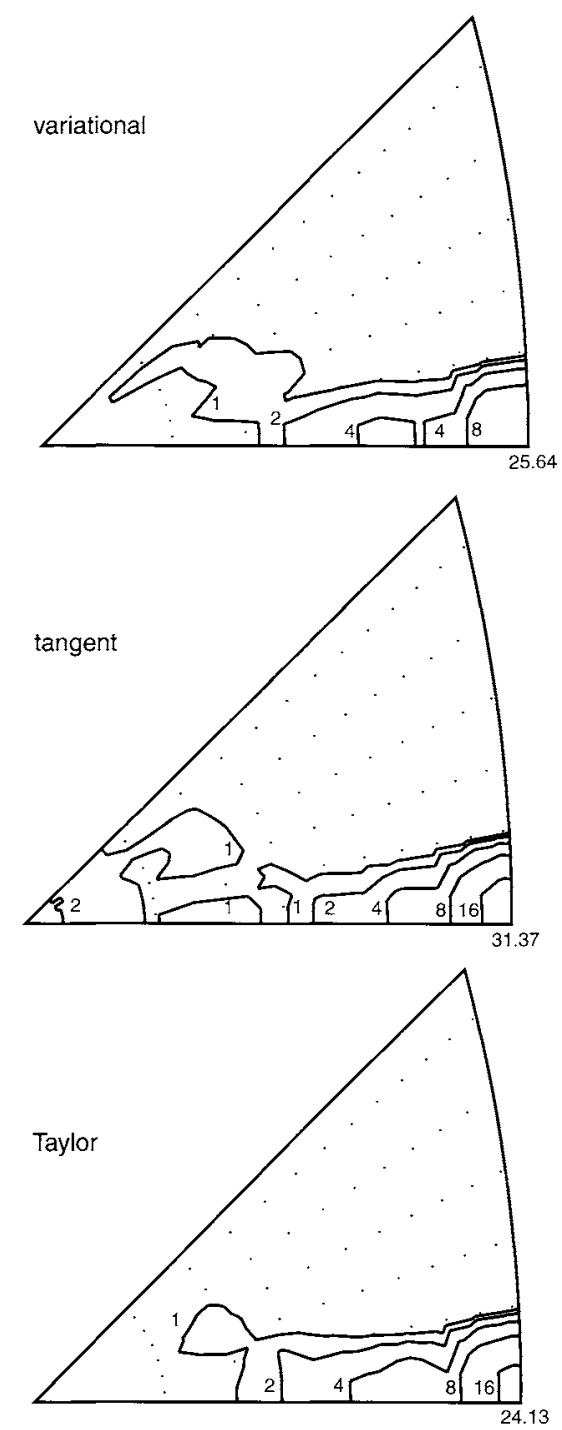

Figure 3. Same as Figure 2 for a uniaxial compression. 
These figures can be compared with Figures 7 and 8 of Wenk et al. [11]. Since $n=9$ was used in that work, comparisons with our room-temperature simulations $(n=10)$ are more appropriate, but it must also be kept in mind that the hardening laws used are different. The agreement is qualitatively good, although the values obtained by Wenk et al. [11] (with hardening) at[100] in both tension and compression with the tangent model appear to be higher.

Another important consideration in comparing the "variational" and "tangent" self-consistent models is the computation time required. Using the same computer, and minimizing the outputs of both programs, it has been found that the variational procedure requires about 6.5 times the computation time of the tangent model. This is still a question of a few tens of minutes on modern computers and is perfectly affordable. On the other hand, this also represents a very significant improvement with respect to earlier implementations (Nebozhyn et al. [6]) of the "variational" method, which used a much slower minimization procedure and would have made the texture evolution computations infeasible. Of course, the Taylor model requires much shorter computation times (tens of seconds) and is rather close to the "variational" model in the present context.

\section{Conclusions}

This paper was concerned with a first implementation of the "variational" homogenization method of deBotton and Ponte Castañeda [2] to predict texture development following the program proposed by Ponte Castaneda [9] for halite polycrystals. As already anticipated by the work of Nebozhyn et al. [6], the predictions of the "variational" method are intermediate between those of the Taylor and "tangent" methods. However, unfortunately, the experimental data that was used here corresponds to a situation where the relevant cubic single crystals are highly isotropic, as expected from the fact that the flow stresses of the various slip families are quite close to each other, even at room temperature (refer to Table 1). Because of this the relative differences in the predictions were rather small. We are presently applying the procedure to more highly anisotropic $\mathrm{HCP}$ polycrystals, where preliminary results show significant differences in the predictions of the various models. These will be reported elsewhere.

\section{Acknowledgments}

YL and PPC acknowledge the support of the NSF through grants CMS-9972234 and INT-97-26521. We are grateful to B. Bacroix for supplying a program to draw texture contour plots and to $\mathrm{O}$. Castelnau for providing a program to compute the "tangent" estimates. 


\section{References}

[1] Carter, N.L. and Heard, H.C. (1970). Am. J. Sci., 269, 193.

[2] deBotton, G. and Ponte Castañeda, P. (1995). Proc. R. Soc. Lond. A, 448, 121.

[3] Heard, H.C. (1972). Am. Geoph. U. Monogr., 16, 191.

[4] Kalidindi, S.R., Bronkhorst, C.A. and Anand, L. (1992). J. Mech. Phys. Solids, 40, 537.

[5] Molinari, A., Canova, G.R. and Ahzi, S. (1987). Acta Metall. Mater., 35, 2983.

[6] Nebozhyn, M.V., Gilormini, P. and Ponte Castañeda, P. (2001). J. Mech. Phys. Solids, 49, 313.

[7] Paquin, A., Berbenni, S., Favier, V., Lemoine, X. and Berveiller, M. (2001).Int. J. Plasticity, 17, 1267.

[8] Ponte Castañeda, P. (1991). J. Mech. Phys. Solids, 39, 45.

[9] Ponte Castañeda, P. (1998). In: Continuum Models and Discrete Systems, Proceedings of the 9th International Symposium, Inan E. and Markov K. Z. [eds.], World Scientific Publishing Co., 228.

[10] Skrotzki, W. and Welch, P. (1983). Tectonophysics, 99, 47.

[11] Wenk, H.R., Canova, G., Molinari, A. and Mecking, H. (1989). Acta Metall., 37, 2017. 